\title{
Análisis del contenido nacional de las exportaciones de manufacturas mexicanas: el caso de la Alianza \\ del Pacífico 2011-2016
}

\section{Analysis of the national content of exports of Mexican manufactures: the case of the Pacific Alliance 2011-2016}

\author{
Oscar Elí Velarde Moreno ${ }^{1}$ \\ Moisés Alejandro Alarcón Osuna ${ }^{2}$ \\ Mónica Blanco Jiménez ${ }^{3}$
}

\begin{abstract}
Resumen
México ha mostrado en los últimos años una elevada actividad exportadora y un cambio en la composición de sus exportaciones, principalmente las manufactureras. No obstante, el problema que enfrenta México es que el crecimiento económico generado por las exportaciones ha sido insuficiente, lo que se asocia con el bajo valor agregado de las exportaciones de la manufactura global (VAEMG). Por tanto, este estudio se realizó buscando determinar el VAEMG de las exportaciones de México a sus socios comerciales de la Alianza del Pacífico. Para esto, se utilizó la metodología del INEGI para calcular el VAEMG y el Sistema
\end{abstract}

de Clasificación Industrial de América del Norte (SCIAN). Los resultados mostraron una disminución en las exportaciones y en su porcentaje de VAEMG, confirmando así que la estrategia basada en impulsar las exportaciones mediante la creación de acuerdos comerciales no está generando el impacto deseado en términos de desarrollo económico.

Palabras clave: Alianza del Pacífico, contenido nacional, exportaciones como motor del crecimiento, exportaciones, tratados comerciales.

Artículo recibido el 17 de enero de 2019 y dictaminado el 18 de mayo de 2019.

1. Universidad Autónoma de Sinaloa. Boulevard Universitarios, Unidad $3 \mathrm{~s} / \mathrm{n}$, Ciudad Universitaria, C. P. 80013. Culiacán, Sinaloa. México. ORcid: http://orcid.org/0000-0002-6017-2598. Correo electrónico: oevelarde@uas.edu.mx

2. Universidad Autónoma de Sinaloa. Boulevard Universitarios, Unidad $3 \mathrm{~s} / \mathrm{n}$, Ciudad Universitaria, C. P. 80013. Culiacán, Sinaloa. México. Orcid: http://orcid.org/0000-0003-3713-0565. Correo electrónico: alarcon93@hotmail.com

3. Universidad Autónoma de Nuevo León. FAcPyA-CEDeEM. Centro de Investigación. Oficina 7. Pedro de Alba s/n. Ciudad Universitaria. San Nicolás de Los Garza, Nuevo León. México. Orcid: http:// orcid.org/0000-0001-7489-4826. Correo electrónico: moniblanco77@hotmail.com 


\begin{abstract}
Mexico has shown in recent years a high export activity and a change in the composition of its exports, mainly manufacturing. However, the problem facing Mexico is that the economic growth generated by exports has been insufficient, which is associated with the low Added Value of Global Manufacturing Exports (AVGME). Therefore, this study was conducted seeking to determine the AVGME of exports from Mexico to its trading partners of the Pacific Alliance. For this, the INEGI methodology
\end{abstract}

was used to calculate the AVGME and the North American Industrial Classification System (NAICS). The results showed a decrease in exports and their percentage of AVGME, confirming that the strategy based on boosting exports through the creation of trade agreements is not generating the desired impact in terms of economic development.

Keywords: Pacific Alliance, national content, export led growth (ELG), exports, trade agreements.

\section{Introducción}

Desde hace algunas décadas ha existido un debate sobre el nexo que vincula al crecimiento económico con el crecimiento de las exportaciones (Giles \& Williams, 2000). De hecho, el crecimiento económico es afectado por muchos factores; no obstante, las exportaciones son uno de los componentes más importantes para fundamentar el crecimiento económico (Medina-Smith, 2000). Por otra parte, este debate no ha sido concluyente sobre las exportaciones como motor del crecimiento económico (Export-Led-Growth) o el crecimiento económico como motor de las exportaciones (Growth-Led-Exports).

Algunos estudios sobre estas temáticas utilizan datos en panel y análisis de correlación. Otros utilizan datos en panel y de corte transversal con métodos de mínimos cuadrados ordinarios (MCO) en una o dos etapas, con y sin efectos fijos y/o aleatorios. Finalmente, otros estudios utilizan técnicas de datos en series de tiempo para examinar la relación causal entre exportaciones y crecimiento económico (Adnan, Wizarat \& Lau, 2013; Yee, 2016).

Bajo este contexto, el modelo ELG es considerado como un importante instrumento para el crecimiento económico en países en vías de desarrollo (Gabrielle, 2004). De acuerdo con Dollar y Kraay (2007) y con Stiglitz (2007), en países en vías de desarrollo el comercio con el exterior tiende a reducir sus niveles de pobreza, tomando como ejemplo a países como China, India, Bangladesh, Malasia y Costa Rica.

De esta forma, México ha mostrado en los últimos años una elevada actividad exportadora y un cambio importante en la composición de las exportaciones, principalmente las de origen manufacturero (Fujii \& Cervantes, 2013), con tecnologías medias y altas (Fujii \& Cervantes, 2013; Levy, 2015). 
No obstante, el problema que enfrenta México es que el crecimiento económico generado por las exportaciones ha sido insuficiente (Fujii \& Cervantes, 2013), ya que a partir de la apertura comercial de México con su entrada al GATT (hoy Organización Mundial de Comercio) en 1988 y posteriormente con la firma del TLCAN en 1994, las exportaciones han crecido más de lo que lo ha hecho el producto interno bruto (PIB).

Algunos autores (Moreno-Brid, Rivas \& Santamaría, 2005) advierten que el modelo no ha funcionado debido a que el crecimiento de las exportaciones también viene acompañado de una fuerte actividad importadora, sobre todo de insumos, y esto dificulta que los encadenamientos productivos se estimulen y generen efectos multiplicadores, lo que finalmente se ve reflejado en exportaciones con bajos niveles de valor agregado.

A pesar de esto, existen estudios que han demostrado que el modelo ELG genera crecimiento en países en vías de desarrollo, especialmente porque son exportadores de materias primas (Yee, 2016). No obstante, en países como México, que exporta principalmente bienes de manufactura media y alta (como automóviles y productos electrónicos), el valor agregado de dichas exportaciones es un factor determinante en cuanto a la generación de crecimiento económico.

Un análisis del valor agregado de las exportaciones mexicanas reveló que en tres de las industrias más representativas los valores agregados de las exportaciones eran inferiores al 50\% (Fuji \& Cervantes, 2013), lo que soporta el argumento de por qué el modelo no ha sido muy efectivo en México; sin embargo, son pocos los estudios que se han realizado al respecto; tampoco se han realizado estudios comparativos para determinar si el tiempo es un factor para que las empresas integren valor agregado nacional, ni se han analizado las exportaciones en términos de valor agregado hacia las diferentes regiones comerciales. Por lo cual, para aportar un mayor entendimiento sobre la eficacia del ELG, el propósito de esta investigación es realizar un estudio de caso para determinar el valor agregado de las exportaciones mexicanas a la Alianza del Pacífico y analizar si se ha generado un crecimiento en este indicador desde la formalización de la Alianza, pues el comercio que ésta origina representa el 44\% del PIB de la región de América Latina y el Caribe (US $\$ 2$ billones en 2014), el 42\% de su población (222 millones) (Molina, Heuser \& Mesquita, 2016). Se analiza a la Alianza del Pacífico con la intención de profundizar en las problemáticas que han impedido que la diversificación de exportaciones 
mexicanas logre detonar el crecimiento económico, pues al parecer es uno de los acuerdos que le genera mayores exportaciones a México después del TLCAN.

El estudio se compone de los siguientes apartados: un marco teórico sobre la relación entre el crecimiento económico y las dinámicas exportadoras, y el contexto de la Alianza del Pacífico, donde se mencionan algunos de los argumentos teóricos de dicho modelo, la relación de las exportaciones mexicanas con el crecimiento del PIB y cuál es la situación de las relaciones comerciales entre México y la Alianza del Pacífico.

En la siguiente sección se describe la metodología empleada para calcular el VAEMG de las exportaciones mexicanas y cómo se empleó la conversión de fracción arancelaria a rama del SCIAN para determinar los VAEMG de las industrias que exportan su producción a los países de la Alianza del Pacífico.

La tercera sección consiste en la presentación de los resultados obtenidos al codificar los VAEMG por ramas de los productos exportados a la Alianza del Pacífico, donde se presenta el valor porcentual del VAEMG y las cantidades en términos monetarios en los años 2011 — que fue la fecha cuando se aprobó la Alianza- y 2016. Y finalmente se cierra con la sección de conclusiones.

\section{El contexto de las exportaciones de México y la balanza comercial con los países de la Alianza del Pacífico}

La economía mexicana es una de las más abiertas a nivel internacional; hasta la fecha de hoy ha firmado 12 acuerdos comerciales con más de 46 países, 32 acuerdos para la promoción y protección recíproca de inversión, y nueve acuerdos económicos complementarios, entre los cuales se encuentra la Alianza del Pacífico; no obstante, esto no ha logrado detonar un mayor crecimiento económico.

A pesar de que las exportaciones de México han crecido de manera importante desde 1988, la balanza comercial de México difícilmente ha registrado superávits comerciales, de hecho desde 1998 a la fecha la suma de cuenta corriente de México con el resto del mundo es deficitaria. Esta condición se observa de mejor manera en la figura 1. 


\section{Figura 1}

Balanza comercial de México 1993-2017

(Miles de dólares)

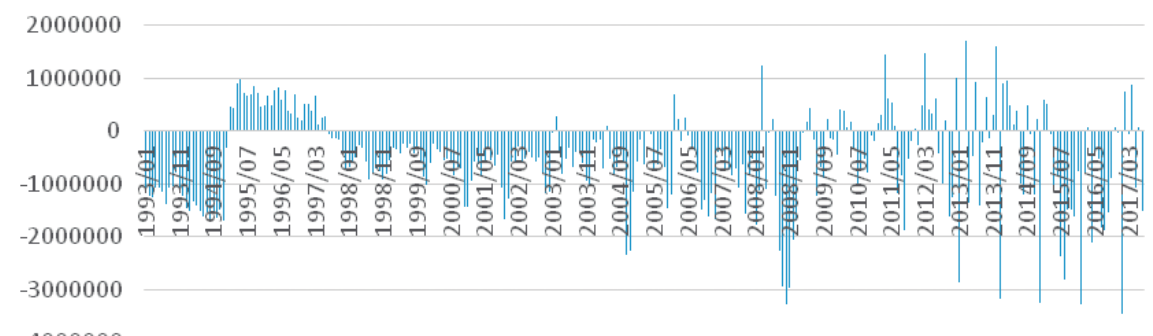

$-4000000$

Fuente: elaboración propia con base en los datos de INEGI-BIE.

La figura 1 muestra que México en los últimos 20 años ha sido un país mayormente deficitario en comercio exterior. Levy (2015) explica que estos déficits se deben principalmente a la industria manufacturera de tecnología intermedia y a productos del sector primario. Como resultado de este comportamiento deficitario, México debe sostener este comportamiento con atracción de flujos financieros del resto del mundo, es decir con inversión extranjera directa e indirecta que puedan sostener el consumo de importación.

Como resultado de este comportamiento, el modelo de crecimiento basado en exportaciones ha logrado capitalizar el TLCAN exportando una gran cantidad de bienes hacia Estados Unidos; no obstante y derivado de esta falta de industrialización, esto ha generado que México tenga que importar bienes manufactureros de tecnología, necesarios para surtir a la cadena de valor de México; esto se muestra de mejor manera con la figura 2. 


\section{Figura 2}

Balanza comercial de México con el mundo por regiones, 1993-2017

(Miles de dólares)

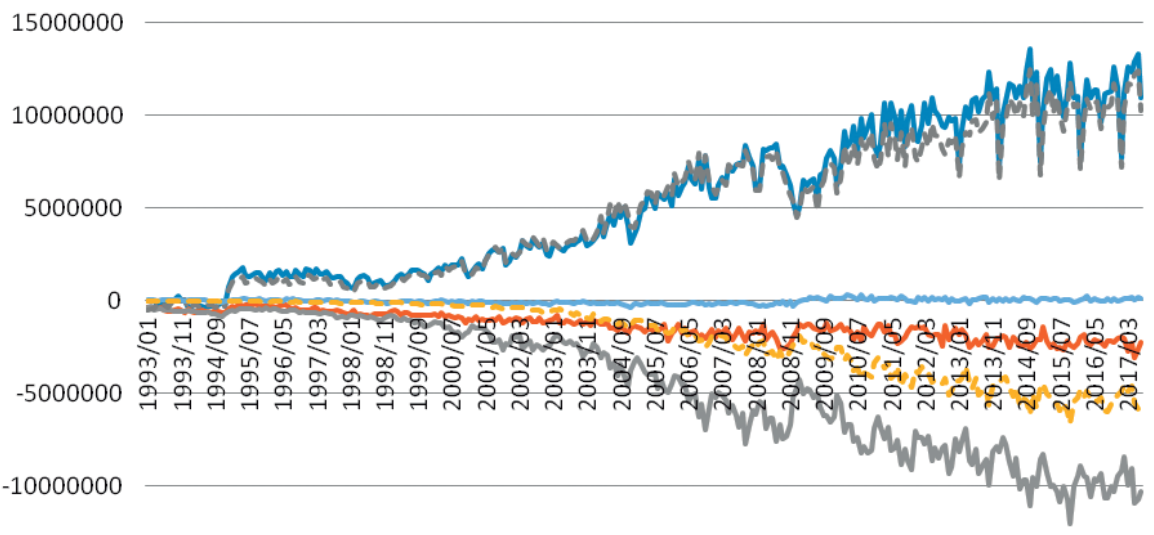

$-15000000$

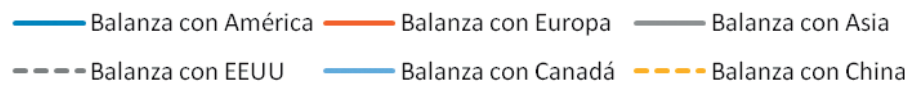

Fuente: elaboración propia con datos de INEGI-BIE.

De la figura 2 se pueden establecer dos cuestiones clave: en primer lugar, como estaba anticipado, la mayor parte del superávit comercial de México viene del comercio con Estados Unidos, pero por otro lado la mayor parte de los déficits comerciales surgen de Asia y Europa. Es decir, que México estratégicamente ha ganado en exportaciones manufactureras de alta tecnología hacia Estados Unidos mediante exportaciones de la industria automotriz y aeronáutica, pero para elaborar estos bienes también ha requerido importar muchos insumos de tecnología intermedia de países como China y otros países de Asia y Europa.

Si bien México ha firmado numerosos acuerdos comerciales, estos podrían no ser de ayuda, puesto que la cadena de valor de México y el modelo de crecimiento basado en exportaciones obliga a que México, para aumentar sus exportaciones, tenga que incrementar forzosamente sus importaciones y anular así los posibles efectos en materia de comercio exterior. Para lograr un crecimiento basado en las exportaciones es necesario desarrollar un esquema de generación de valor agregado y mayor contenido nacional en las exportaciones, lo que se podría aprovechar mediante nuevos acuerdos comerciales con 
países que también están en vías de desarrollo, tal es el caso de los países como Perú, Colombia y Chile, que junto con México forman la Alianza del Pacífico.

El acuerdo con los países de la Alianza del Pacífico se oficializó a partir del año 2011, desde entonces México ha mantenido un superávit comercial con sus contrapartes de Perú, Colombia y Chile. Sin embargo, es difícil saber el beneficio que se tiene de estos superávits ya que muchos de los productos exportados podrían ser de bajo valor agregado, de ahí la importancia de realizar un análisis de las exportaciones calculando dicho indicador. La balanza comercial de México con los países de la Alianza del Pacífico se presenta en la figura 3.

\section{Figura 3}

Balanza comercial de México con los países de la Alianza del Pacífico, 2011-2017 (Miles de dólares)

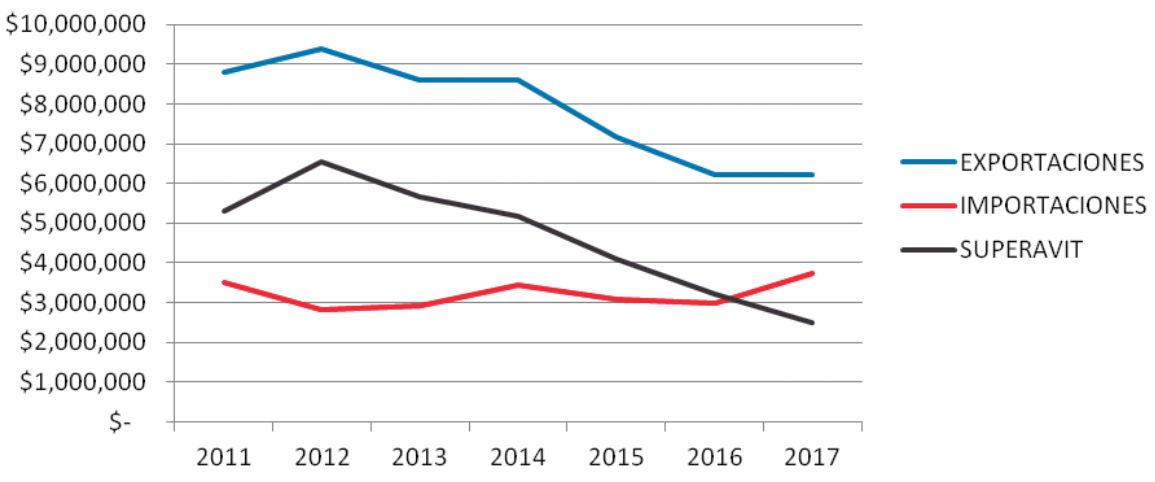

Fuente: elaboración propia con datos de INEGI y Banxico, 2017.

\section{El modelo de crecimiento basado en exportaciones}

De acuerdo con Fujii y Cervantes (2013), algunos de los argumentos en pro del modelo ELG son:

- Que la competencia a nivel internacional puede estimular una mayor eficiencia del aparato productivo (Bhagwati \& Srinivasan, 1979; Feder, 1983; Kohli \& Singh, 1989; Krueger, 1980).

- Las exportaciones conducen a la especialización, lo que a su vez conduce a las economías de escala (Helpman \& Krugman, 1985).

- Las empresas orientadas a la exportación tienden a ser tecnológicamente más avanzadas y estimulan un progreso técnico y derramas en el resto de la economía (Grossman \& Helpman, 1991). 
- Las exportaciones hacen posible superar la restricción externa al crecimiento (Thirlwall, 1979).

En este sentido y con los argumentos señalados, el modelo ELG propone una relación positiva entre el nivel de exportaciones y el crecimiento económico (Yee, 2016). No obstante, se plantea la posibilidad de que países que se concentran en la producción manufacturera crecerán de manera más rápida que aquellos que se concentran en la producción de bienes primarios (Hausmann, Hwang \& Rodrik, 2007). De allí la idea de que las exportaciones con mayor contenido tecnológico se beneficiarán más de las externalidades positivas que detonen el crecimiento económico.

De esta forma, Helpman y Krugman (1985) proponen que el crecimiento en las exportaciones determina el crecimiento económico mediante economías de escala, especialización en la producción y diseminación del conocimiento. Bhagwati (1988) señala que además de lo anterior, el crecimiento en las exportaciones promueve el crecimiento económico, y que el crecimiento económico a su vez detona la formación de habilidades y el progreso tecnológico.

Finalmente, el modelo ELG detona la eficiencia económica a través de una mejor asignación de recursos y promoción del crecimiento económico en el largo plazo (Gokmenoglu, Sehnaz \& Taspinar, 2015), esto debido a que orienta a las empresas a especializarse en la producción de bienes de exportación que llevarán a un aumento en el nivel de productividad, donde los empleados mejor capacitados estarán ubicados en el sector exportador.

No obstante, en el caso de México la capacidad de las exportaciones para dinamizar el producto ha sido cada vez más débil, pues como muestra la figura 1 , la brecha entre ambos se ha ampliado en los últimos años y en particular a partir de 1988 .

\section{Metodología}

La literatura empírica sobre estos estudios se puede dividir en tres partes (Adnan, Wizarat \& Lau, 2013; Yee, 2016): la primera, que utiliza métodos de datos en panel y análisis de correlación para probar la hipótesis "Export-LedGrowth" (ELG). Un segundo grupo de estudios, que utiliza datos en panel y de corte transversal con métodos de mínimos cuadrados ordinarios (MCO) en una o dos etapas, con y sin efectos fijos y/o aleatorios. Finalmente, un tercer grupo 
de estudios que utiliza técnicas de datos en series de tiempo para examinar la relación causal entre exportaciones y crecimiento económico.

Para este caso se utiliza una metodología similar a la empleada por Fujii y Cervantes (2013), donde los autores calculan el contenido nacional en las exportaciones manufactureras. Si bien en este trabajo se retoma la idea de calcular el valor agregado de las exportaciones de la industria manufacturera como lo hacen Fujii y Cervantes (2013), este estudio se diferencia del anterior en la medida en que aquí señalamos cuál es el valor agregado de las exportaciones manufactureras, haciendo un desglose por rama industrial y subsector de SCIAN, tomando al final los más importantes para realizar un comparativo del año 2011, que es la fecha de la firma, contra los de 2016 y determinar qué tanto mejoraron las exportaciones en términos de valor agregado.

No obstante, la metodología empleada en este estudio mide el porcentaje del valor agregado de las diferentes subsectores del sciAN acorde con la contenida en el Instituto Nacional de Estadística, Geografía e Informática (INEGI, 2017), dentro de la cual se consideran las empresas que cumplen con los siguientes criterios:

- Empresas cuyos insumos provienen del exterior principalmente y su producción se destina a la exportación.

- Empresas que tienen una participación mayoritaria de capital extranjero.

- Empresas exportadoras de bienes intermedios.

El valor agregado de exportación de la manufactura global puede obtenerse de dos maneras:

$$
\text { VAEMG }=\text { VABPMG }+ \text { INpmg }
$$

Donde VAEMG es el valor agregado de exportación de la manufactura global, VABPMG el valor agregado bruto de la producción manufacturera global y INpmg el insumo nacional de la producción manufacturera global.

$$
\mathrm{VAEMG}=\mathrm{XPMG}-\mathrm{Mpmg}
$$

Donde VAEMg es el valor agregado de exportación de la manufactura global, XPMG exportaciones de la producción manufacturera global y INpmg es importaciones de la producción manufacturera global. 
Para medir el impacto del VAEMG, el INEGI (2017) ha elaborado algunos indicadores donde se incorporan estimaciones de la contribución por subsector de actividad, dicho subsector de actividad pertenece a la clasificación del SCIAN. A este indicador se le denomina VAEMG como proporción manufacturera global, y se obtiene de la siguiente fórmula:

$$
\operatorname{VAEMG}(\% \mathrm{PM})=[\mathrm{VAEMG} / \mathrm{PMG}]{ }^{*} 100
$$

Donde VAEMg (\%PM) es el valor agregado como proporción de las exportaciones de la industria manufacturera global, VAEMG el valor agregado de exportación del subsector de la rama económica de la manufactura global y PMG la producción manufacturera global.

Para conocer la cantidad de bienes exportados por México a los países de la Alianza del Pacífico se extrajeron los datos de las exportaciones en dólares por producto de acuerdo con su fracción arancelaria con base en los datos del INEGI del año 2011 y el año 2016.

Los datos de la tabla 1, referentes a la exportación de bienes a la Alianza del Pacífico estaban clasificados de acuerdo con la fracción arancelaria; debido a que el porcentaje de valor agregado de las exportaciones se clasifica en los sectores, subsectores y ramas del SCIAN, fue necesario identificar qué fracciones se agrupan en las subramas de dicha clasificación mediante una tabla denominada TIGIE-SCIAN (INEGI, 2016).

\section{Tabla 1}

Conversión de fracción arancelaria a código SCIAN

\begin{tabular}{c|c|l|c}
\hline Fracción & $\begin{array}{c}\text { Exportación en } \\
\text { dólares }\end{array}$ & \multicolumn{1}{|c|}{ Descripción de la fracción arancelaria } & $\begin{array}{c}\text { Clase de } \\
\text { actividad SCIAN }\end{array}$ \\
\hline 87032301 & $\$ 620^{\prime} 482,165.00$ & $\begin{array}{l}\text { De cilindrada superior a } 1,500 \mathrm{~cm}^{3} \\
\text { pero inferior o igual a 3,000 } \mathrm{cm}^{3}\end{array}$ & $336-110$ \\
\hline 87012001 & $\begin{array}{l}\text { Tractores de carretera para } \\
\text { semirremolques, excepto lo } \\
\text { comprendido en la fracción } 87012002\end{array}$ & $336-120$ \\
\hline 87163102 & $\$ 2^{\prime} 391,222.00$ & $\begin{array}{l}\text { Tipo tanques de acero, incluso } \\
\text { criogénicos o tolvas }\end{array}$ & $336-210$ \\
\hline 85122099 & $\$ 1^{\prime} 164,783.00$ & Los demás & $336-320$ \\
\hline
\end{tabular}

Fuente: elaboración propia con base en la tabla TIGIE-SCIAN y datos del INEGI. 
Para presentar la información de una manera gráfica y evitar presentar una gran cantidad de información, los valores se agruparon hasta el tercer dígito de dicha clasificación, dado que el número de subramas era muy amplio como para elaborar un análisis gráfico del valor agregado.

El sCIAN es una clasificación jerárquica que tiene cinco niveles. El primer nivel es el sector y corresponde a los dos primeros números del código; en el caso del sector de la industria manufacturera, los números abarcan del 31 al 33. El subsector es un nivel más específico que se desprende del sector, y se representa por los tres primeros dígitos del código SCIAN; un ejemplo sería el subsector de fabricación de equipo de transporte, cuyo código de representación es 336. Los siguientes niveles son la rama, subrama y clase de actividad, siendo el último el más específico; un ejemplo de esta clasificación puede observarse en la tabla 2. Como ya se mencionó, debido a que existen demasiadas clases de actividad, ramas y subramas, la información presentada en esta investigación se realizó a partir del subsector.

Tabla 2

Niveles del código SCIAN

\begin{tabular}{c|c|l}
\hline Nivel & Código & Ejemplos de categoría \\
\hline Sector & $31-33$ & Industrias manufactureras \\
\hline Subsector & 336 & Fabricación de equipo de transporte \\
\hline Rama & 3361 & Fabricación de automóviles y camiones \\
\hline Subrama & 33611 & Fabricación de automóviles y camionetas \\
\hline Clase de actividad & 336110 & Fabricación de automóviles y camionetas \\
\hline
\end{tabular}

Fuente: elaboración propia con base en la tabla TIGIE-SCIAN.

\section{Análisis de las exportaciones de México a la Alianza del Pacífico}

En el año 2011 las exportaciones de México a los países de la Alianza del Pacífico alcanzaron los 8,796 millones de dólares de acuerdo con las estadísticas de INEGI; el 31\% de esas exportaciones correspondió al sector de fabricación de equipo de transporte; la fabricación de equipos de comunicación, medición y de otros tipos de componentes y accesorios electrónicos representó el 19\%; el 15\% fueron productos de la industria química; $9 \%$ de industrias metálicas básicas. Cabe señalar además que cerca del $98 \%$ del total de las exportaciones son manufacturas, mientras que el restante $2 \%$ corresponde a la industria no 
manufacturera. La figura 4 muestra la división de las exportaciones por rama del sciAn en el año 2011.

\section{Figura 4}

Porcentaje de exportaciones por subsector SCIAN, 2011

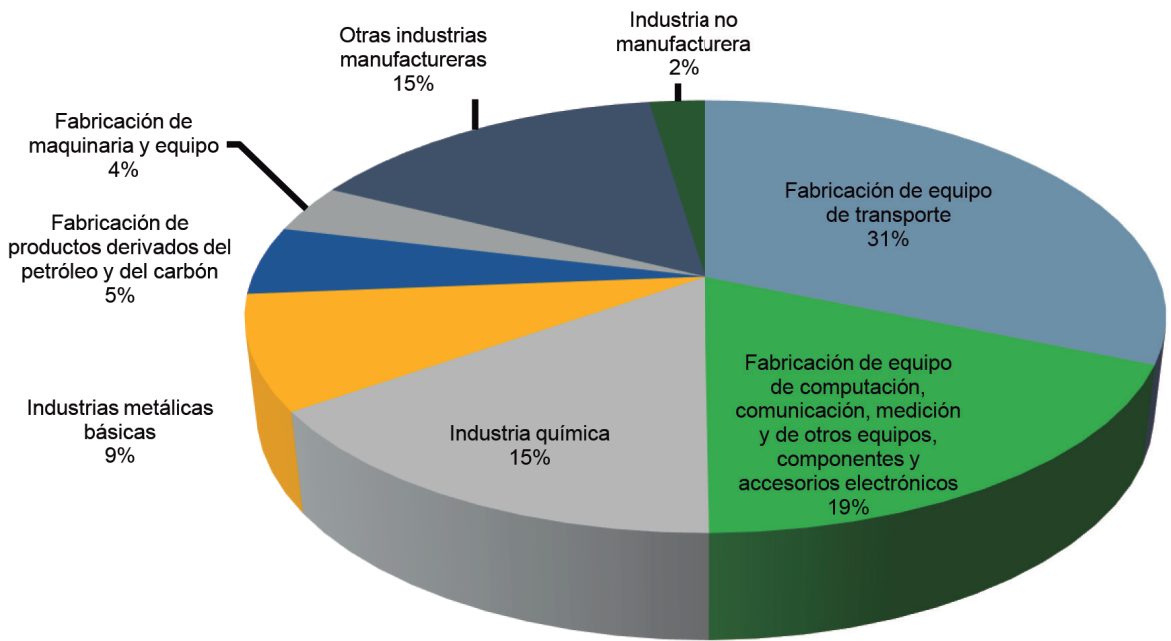

Fuente: elaboración propia con base en datos del INEGI (2016).

En el año 2016 la composición de las exportaciones de México a los países de la Alianza del Pacífico tuvo un cambio significativo respecto a 2011, ya que primeramente la cantidad total de exportaciones se redujo a 6,214 millones de dólares de acuerdo con las estadísticas del INEGI, donde las exportaciones de las manufacturas correspondientes al sector de la industria del transporte se redujeron al 21\% del total; la fabricación de equipos de comunicación, medición y de otros tipos de componentes y accesorios electrónicos se elevó a $21 \%$, y la industria química a $18 \%$, mientras que las industrias metálicas básicas representaron sólo $5 \%$. La participación de las exportaciones no manufactureras también tuvo un ligero retroceso ya que sólo representaron el $1 \%$ de las exportaciones totales, contra $99 \%$ que representaron las de manufacturas. La figura 5 muestra la proporción de las exportaciones en términos porcentuales por subsector del sciAN en el año 2016. 


\section{Figura 5}

Porcentaje de exportaciones por subsector SCIAN, 2016

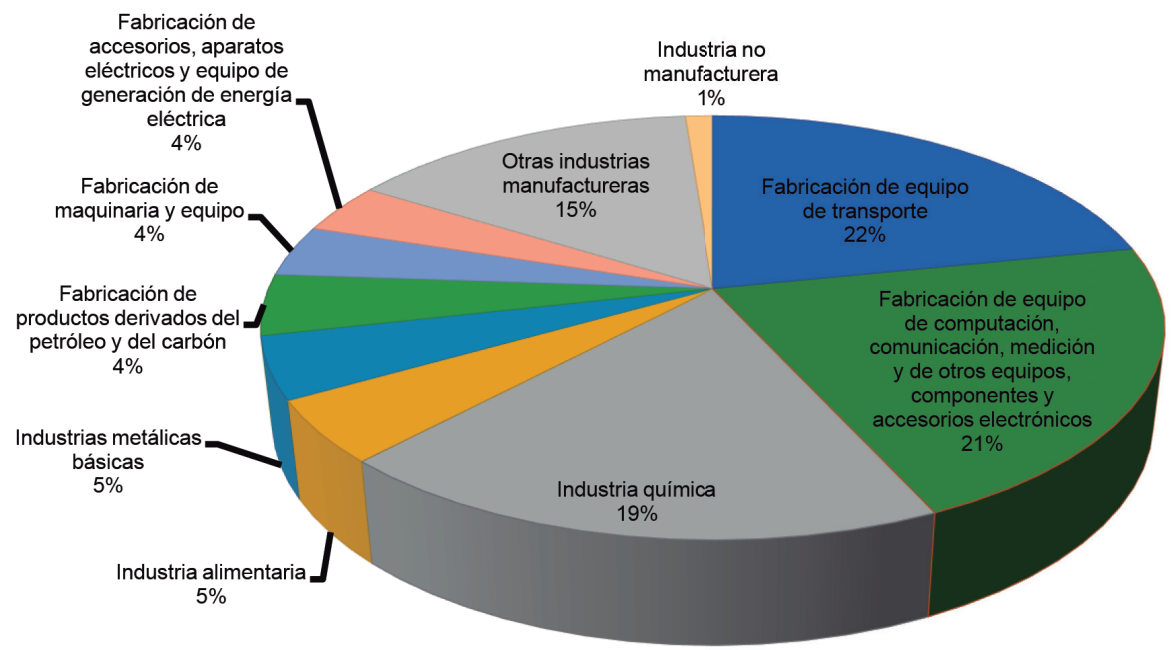

Fuente: elaboración propia con base en datos del INEGI (2016).

En 2011 México realizó exportaciones de equipo de transporte a sus contrapartes de la Alianza del Pacífico por más de 2,762 millones de dólares. Esta industria, de acuerdo con los datos del INEGI tiene un $45 \%$ de valor agregado de la manufactura global, por lo cual se estima que en términos de valor agregado las exportaciones de este sector fueron de 1,229 millones de dólares. La industria de fabricación de equipo de computación, comunicación, medición y de otros equipos, componentes y accesorios electrónicos fue la segunda más importante en el valor de sus exportaciones; sin embargo, en 2011 su VAEMG se estimaba en sólo $17 \%$ por lo que en términos de valor agregado sólo se exportaron cerca de 270 millones de dólares.

La industria metálica básica es una de las que tiene un mayor VAEMG en términos porcentuales, ya que cuenta con el $69 \%$, de tal manera que de los 749 millones de dólares exportados, 515 millones de dólares corresponden al valor agregado. El cálculo del valor agregado del total de las exportaciones a los países de la Alianza del Pacífico en 2011 fue del 39\% por lo que se puede estimar que de los 8,796 millones de dólares exportados, sólo 3,444 millones de dólares representaron el valor agregado nacional. La proporción del VAEMG 
de las exportaciones mexicanas a los países de la Alianza del Pacífico en el año 2011 puede observarse en la tabla 3.

\section{Tabla 3}

VAemg de las exportaciones mexicanas a la Alianza del Pacífico, 2011

(En dólares)

\begin{tabular}{c|l|c|c|c}
\hline $\begin{array}{c}\text { Código } \\
\text { SCIAN }\end{array}$ & \multicolumn{1}{|c|}{ Rama del SCIAN } & \multicolumn{1}{|c|}{$\begin{array}{c}\text { Valor de las } \\
\text { exportaciones }\end{array}$} & VAEMG\% & $\begin{array}{c}\text { Exportaciones en } \\
\text { VAEMG }\end{array}$ \\
\hline 336 & $\begin{array}{l}\text { Fabricación de equipo } \\
\text { de transporte }\end{array}$ & $\$ 2,762^{\prime} 959,998.00$ & $45 \%$ & $\$ 1,229^{\prime} 517,199.11$ \\
\hline & $\begin{array}{l}\text { Fabricación de equipo } \\
\text { de computación, } \\
\text { comunicación, } \\
\text { medición y de otros } \\
\text { equipos, componentes y } \\
334\end{array}$ & $\begin{array}{l}\$ 1,622^{\prime} 346,215.00 \\
\text { accesorios electrónicos }\end{array}$ & $17 \%$ & $\$ 270^{\prime} 931,817.91$ \\
\hline 325 & Industria química & $\$ 1,348^{\prime} 668,543.00$ & $35 \%$ & $\$ 472^{\prime} 033,990.05$ \\
\hline 331 & $\begin{array}{l}\text { Industrias metálicas } \\
\text { básicas }\end{array}$ & $\$ 749^{\prime} 024,975.00$ & $69 \%$ & $\$ 515^{\prime} 329,182.80$ \\
\hline 324 & $\begin{array}{l}\text { Fabricación de } \\
\text { productos derivados del } \\
\text { petróleo y del carbón }\end{array}$ & $\$ 449^{\prime} 815,497.00$ & $35 \%$ & $\$ 156^{\prime} 985,608.45$ \\
\hline 333 & $\begin{array}{l}\text { Fabricación de } \\
\text { maquinaria y equipo }\end{array}$ & $\$ 316^{\prime} 611,649.00$ & $34 \%$ & $\$ 106^{\prime} 381,514.06$ \\
\hline 339 & $\begin{array}{l}\text { Otras industrias } \\
\text { manufactureras }\end{array}$ & $\$ 1,339^{\prime} 959,220.00$ & $37 \%$ & $\$ 490^{\prime} 489,418.79$ \\
\hline 111 & $\begin{array}{l}\text { Industria no } \\
\text { manufacturera }\end{array}$ & $\$ 206^{\prime} 643,384.00$ & $100 \%$ & $\$ 206^{\prime} 643,384.00$ \\
\hline & Valor agregado total & $\$ 8,796^{\prime} 029,481.00$ & $39 \%$ & $\$ 3,448^{\prime} 312,115.17$ \\
\hline
\end{tabular}

Fuente: elaboración propia con base en datos del INEGI (2017).

En el año 2016 las exportaciones mexicanas de equipo de transporte disminuyeron en $50 \%$ respecto a 2011, y el porcentaje del valor agregado se mantuvo en $45 \%$ por lo cual el VAEMG exportado de esta industria fue de 611 millones de dólares. Las exportaciones de equipos de computación, comunicación, medición, componentes y accesorios electrónicos disminuyó pero el VAEMG de esta industria aumentó al 22\% lo que propició un mayor valor agregado respecto a 2011 de 292 millones de dólares. La industria química redujo ligeramente 
las exportaciones a 1,167 millones de dólares e incrementó el porcentaje del valor agregado al 37\% para un VAEMG total de 428 millones de dólares.

Las exportaciones de las manufacturas metálicas básicas disminuyeron más del 50\%, lo cual posiblemente se relaciona con la disminución de exportaciones de la industria del transporte, por lo cual su VAEMG total fue de 187 millones de dólares. Respecto al valor agregado total de las exportaciones a los países de la Alianza del Pacífico en términos porcentuales, hubo una ligera disminución respecto a 2011, siendo de $37.6 \%$ y representa un valor agregado total de 2,335 millones de dólares. La proporción del VAEMG de las exportaciones mexicanas a los países de la Alianza del Pacífico en el año 2016 puede observarse en la tabla 4.

\section{Tabla 4}

VAemg de las exportaciones mexicanas a la Alianza del Pacífico, 2016 (En dólares)

\begin{tabular}{|c|c|c|c|c|}
\hline $\begin{array}{l}\text { Código } \\
\text { SCIAN }\end{array}$ & Rama del SCIAN & $\begin{array}{l}\text { Valor de las } \\
\text { exportaciones }\end{array}$ & VAEMG \% & VAEMG \\
\hline 336 & $\begin{array}{l}\text { Fabricación de equipo de } \\
\text { transporte }\end{array}$ & $\$ 1,353 ’ 873,491.00$ & $45 \%$ & $\$ 611^{\prime} 950,817.93$ \\
\hline 334 & $\begin{array}{l}\text { Fabricación de equipo } \\
\text { de computación, } \\
\text { comunicación, medición } \\
\text { y de otros equipos, } \\
\text { componentes y accesorios } \\
\text { electrónicos }\end{array}$ & $\$ 1,330 ’ 076,406.00$ & $22 \%$ & $\$ 292 ' 616,809.32$ \\
\hline 325 & Industria química & $\$ 1,167 ’ 450,817.00$ & $37 \%$ & $\$ 428^{\prime} 454,449.84$ \\
\hline 311 & Industria alimentaria & $\$ 295^{\prime} 968,531.00$ & $58 \%$ & $\$ 171^{\prime} 069,810.92$ \\
\hline 331 & $\begin{array}{l}\text { Industrias metálicas } \\
\text { básicas }\end{array}$ & $\$ 293^{\prime} 060,256.00$ & $64 \%$ & $\$ 187 ’ 265,503.58$ \\
\hline 324 & $\begin{array}{l}\text { Fabricación de productos } \\
\text { derivados del petróleo y del } \\
\text { carbón }\end{array}$ & $\$ 288^{\prime} 988,153.00$ & $37 \%$ & $\$ 107 ’ 214,604.76$ \\
\hline 333 & $\begin{array}{l}\text { Fabricación de maquinaria } \\
\text { y equipo }\end{array}$ & $\$ 243^{\prime} 502,703.00$ & $31 \%$ & $\$ 74 ’ 998,832.52$ \\
\hline 335 & $\begin{array}{l}\text { Fabricación de accesorios, } \\
\text { aparatos eléctricos y } \\
\text { equipo de generación de } \\
\text { energía eléctrica }\end{array}$ & $\$ 238 ’ 518,383.00$ & $36 \%$ & $\$ 85^{\prime} 389,581.11$ \\
\hline
\end{tabular}


Oscar Elí Velarde Moreno, Moisés Alejandro Alarcón Osuna y Mónica Blanco Jiménez

\begin{tabular}{c|l|c|c|c}
\hline $\begin{array}{c}\text { Código } \\
\text { SCIAN }\end{array}$ & \multicolumn{1}{|c|}{ Rama del SCIAN } & $\begin{array}{c}\text { Valor de las } \\
\text { exportaciones }\end{array}$ & VAEMG $\%$ & VAEMG \\
\hline & $\begin{array}{l}\text { Otras industrias } \\
\text { manufactureras }\end{array}$ & $\$ 928,397,821.00$ & $32 \%$ & $\$ 301^{\prime} 665,715.52$ \\
\hline 111 & $\begin{array}{l}\text { Industria no } \\
\text { manufacturera }\end{array}$ & $\$ 74,863,975.00$ & $100 \%$ & $\$ 74^{\prime} 863,975.00$ \\
\hline & Valor agregado total & $\$ 6,214^{\prime} 700,536.00$ & $37.6 \%$ & $\$ 2,335^{\prime} 490,100.52$ \\
\hline
\end{tabular}

Fuente: elaboración propia con base en datos del INEGI (2017).

A pesar de tener un contenido inferior al 50\% del VAEMG, la industria del transporte es la que generó la principal aportación de valor agregado, representando el $36 \%$ del total, seguido de las manufacturas de la industria metálica básica que representaron $15 \%$, por encima de la industria química y otras industrias manufactureras, ambas con $14 \%$. La industria de electrónicos y aparatos eléctricos, pese a ser la segunda más importante en cantidad de exportaciones, sólo representó $8 \%$, apenas arriba de la industria no manufacturera, que obtuvo $6 \%$. Los resultados pueden observarse en la tabla 5.

Tabla 5

Aportación al VAEMG en dólares y términos porcentuales por rama del SCIAN en el año 2011

\begin{tabular}{c|l|c|c}
\hline $\begin{array}{c}\text { Código } \\
\text { SCIAN }\end{array}$ & \multicolumn{1}{|c|}{ Rama del SCIAN } & \multicolumn{1}{|c}{ VAEMG } & $\begin{array}{c}\text { Aportación al } \\
\text { VAEMG }\end{array}$ \\
\hline 336 & Fabricación de equipo de transporte & $\$ 1,229^{\prime} 517,199.11$ & $36 \%$ \\
\hline 331 & Industrias metálicas básicas & $\$ 515^{\prime} 329,182.80$ & $15 \%$ \\
\hline 339 & Otras industrias manufactureras & $\$ 490^{\prime} 489,418.79$ & $14 \%$ \\
\hline 325 & Industria química & $\$ 472^{\prime} 033,990.05$ & $14 \%$ \\
\hline & $\begin{array}{l}\text { Fabricación de equipo de computación, } \\
\text { comunicación, medición y de otros } \\
\text { equipos, componentes y accesorios } \\
\text { electrónicos }\end{array}$ & $\$ 270^{\prime} 931,817.91$ & $8 \%$ \\
\hline 111 & Industria no manufacturera & $\$ 206^{\prime} 643,384.00$ & $6 \%$ \\
\hline 324 & $\begin{array}{l}\text { Fabricación de productos derivados del } \\
\text { petróleo y del carbón }\end{array}$ & $\$ 156^{\prime} 985,608.45$ & $5 \%$ \\
\hline 333 & Fabricación de maquinaria y equipo & $\$ 106^{\prime} 381,514.06$ & $3 \%$ \\
\hline & Valor agregado total & $\$ 3,448^{\prime} 312,115.17$ & $100 \%$ \\
\hline
\end{tabular}

Fuente: elaboración propia con base en datos del INEGI (2017). 
En el año 2016 la aportación de la industria de transporte al VAEMG se vio reducida a un $26 \%$ a pesar de mantener el mismo porcentaje de valor agregado, esto debido a la gran disminución de las exportaciones de estas manufacturas a estos países. Las exportaciones de manufacturas de la industria química y de equipo de computación, comunicación, medición y de otros equipos, componentes y accesorios electrónicos aumentaron su participación principalmente por la disminución de las exportaciones totales, ya que en términos de valor agregado sus valores disminuyeron respecto a 2011. Los resultados pueden observarse en la tabla 6.

Tabla 6

Aportación al VAEMg en dólares y términos porcentuales por rama del SCIAN en el año 2016

\begin{tabular}{|c|c|c|c|}
\hline $\begin{array}{l}\text { Código } \\
\text { SCIAN }\end{array}$ & Rama del SCIAN & VAEMG & $\begin{array}{c}\text { Aportación al } \\
\text { VAEMG \% }\end{array}$ \\
\hline 336 & Fabricación de equipo de transporte & $\$ 611 ’ 950,817.93$ & $26 \%$ \\
\hline \multirow[t]{3}{*}{325} & Industria química & $\$ 428 ’ 454,449.84$ & $18 \%$ \\
\hline & Otras industrias manufactureras & $\$ 301^{\prime} 665,715.52$ & $13 \%$ \\
\hline & $\begin{array}{l}\text { Fabricación de equipo de computación, } \\
\text { comunicación, medición y de otros } \\
\text { equipos, componentes y accesorios } \\
\text { electrónicos }\end{array}$ & $\$ 292 ’ 616,809.32$ & $13 \%$ \\
\hline 331 & Industrias metálicas básicas & $\$ 187 ’ 265,503.58$ & $8 \%$ \\
\hline 311 & Industria alimentaria & $\$ 171^{\prime} 069,810.92$ & $7 \%$ \\
\hline 324 & $\begin{array}{l}\text { Fabricación de productos derivados del } \\
\text { petróleo y del carbón }\end{array}$ & $\$ 107 ’ 214,604.76$ & $5 \%$ \\
\hline 335 & $\begin{array}{l}\text { Fabricación de accesorios, aparatos } \\
\text { eléctricos y equipo de generación de } \\
\text { energía eléctrica }\end{array}$ & $\$ 85^{\prime} 389,581.11$ & $4 \%$ \\
\hline 333 & Fabricación de maquinaria y equipo & $\$ 74 ’ 998,832.52$ & $3 \%$ \\
\hline \multirow[t]{2}{*}{111} & Industria no manufacturera & $\$ 74 ’ 863,975.00$ & $3 \%$ \\
\hline & Valor agregado total & $\$ 2,335^{\prime} 490,100.52$ & $100 \%$ \\
\hline
\end{tabular}

Fuente: elaboración propia con base en datos del INEGI (2017).

\section{Conclusiones}

Los resultados obtenidos en esta investigación son muy similares a los encontrados por Fujii y Cervantes (2013), aunque este estudio permite profundizar 
más en otras industrias que tienen una alta participación en el VAEMG, como es el caso de las industrias metálicas básicas y la industria química, que a pesar de exportarse en volúmenes inferiores que la industria eléctrica y electrónica, aportan un mayor valor agregado. Sin embargo, los patrones de exportación de México son muy similares a los presentados con sus socios comerciales del TLCAN, ya que de igual manera la fabricación de equipo de transporte es el principal subsector de exportaciones a los países de la Alianza del Pacífico.

Otra aportación del estudio es la oportunidad de comparar las exportaciones en los años 2011 y 2016, cuando el volumen de las exportaciones de México a estos destinos se ha visto reducido; dicha reducción es ocasionada principalmente por la disminución de exportaciones de equipo de transporte, lo cual también afectó al VAEMG ya que es uno de los subsectores con mayor valor agregado, con el $45 \%$. Aunque cabe señalar que el porcentaje de VAEMG de este subsector no presentó mejorías a pesar de que en México se han creado indicadores y políticas para incrementar la participación de los proveedores locales en las exportaciones de la industria automotriz.

Tampoco ha habido aumentos considerables en las exportaciones de productos clasificados en otras ramas de SCIAN que pudieran mitigar dicha reducción de las exportaciones de autotransporte. Adicionalmente, los niveles de valor agregado en las exportaciones, al ser inferiores al 40\%, refuerzan la hipótesis de que México es un país que depende de las importaciones para exportar; además el porcentaje de VAEMG no aumentó en 2016, sino que por el contrario, se redujo en casi un $2 \%$, que a pesar de no ser tan considerable demuestra un estancamiento en el valor agregado como porcentaje del total de las exportaciones. En términos de VAEMG exportado, la reducción fue más significativa con $32 \%$.

Dentro de lo positivo, se presentaron mejorías en otros subsectores principales en términos de valor agregado, como el caso de la industria de electrónicos, cuyo aumento fue de $5 \%$, y la industria química con $2 \%$. A pesar de esto su valor agregado sigue siendo relativamente bajo con 22 y $37 \%$, respectivamente, lo que se traduce en una pobre aportación al VAEMG en términos porcentuales.

El estancamiento en los valores del VAEMG sugiere una problemática en cuanto a la integración de proveedores locales en las cadenas productivas, y a pesar del transcurso de los años no se ve reflejada una mejora significativa en los porcentajes de valor agregado de las exportaciones. 
Por lo tanto, se puede concluir que la fabricación de transporte representa el subsector principal de exportaciones de México a la Alianza del Pacífico y debido a su relativamente alto VAEMG la menor variación que sufra dicha industria afecta significativamente a las exportaciones, al VAEMG y por supuesto al crecimiento económico del país.

Además, los valores obtenidos demuestran que la firma del acuerdo no ha brindado los resultados esperados en lo que respecta a impulsar el comercio exterior, ya que como ya se mencionó, desde su elaboración y aceptación en el año 2011, el volumen de las exportaciones ha disminuido hasta un 30\% en el periodo de 2016, impactado directamente por el subsector autotransporte. Una posible causa de esta situación es que quizá el mercado del TLCAN sea más atractivo para las grandes empresas multinacionales, ocasionando una redirección de las exportaciones, o quizá debido a que la competencia dentro de los países de destino ha reducido la demanda de estos bienes. Independientemente de la razón, esta disminución de las exportaciones y sobre todo el VAEMG podría significar una disminución en crecimiento económico de México.

Respecto al modelo de ELG, no se puede negar que ha impactado positivamente en términos generales al desarrollo económico en México y otros países, aunque existen diversas oportunidades que se pueden mejorar para sacarle un mayor provecho a los acuerdos comerciales con los que México cuenta y así poder diversificar las exportaciones.

Si bien es cierto que México mantiene sus niveles de superávit comercial con sus socios comerciales de la Alianza del Pacífico, el bajo valor agregado de sus exportaciones crea una duda respecto a la relevancia de dicho indicador de la balanza comercial. Sin embargo, la constante amenaza de la renegociación de los términos del TLCAN hace que otros acuerdos como la Alianza del Pacífico se mantengan relevantes como una oportunidad para diversificar las exportaciones.

Por estas razones es recomendable fomentar el desarrollo de proveedores locales haciendo hincapié en la integración de pymes a las cadenas de valor con el objetivo de incrementar los VAEMG y que las exportaciones generen un impacto directo en el PIB del país. También es importante que se generen estrategias para la diversificación de exportaciones a otros países con los que se tienen acuerdos, como es el caso de los socios comerciales de la Alianza del Pacífico, incluso pymes que puedan exportar directamente sin la necesidad de entrar en las cadenas productivas de multinacionales. Sólo así esta estrategia de ELG podrá generar los beneficios esperados, de otro modo los resultados 
podrían seguir siendo ineficientes y sumar a México en una larga lista de debates sobre la importancia de las exportaciones para el crecimiento económico.

\section{Referencias bibliográficas}

Adnan, Q., Wizarat, S., \& Lau, W. (2013). Trade-led growth hypothesis: An empirical analysis of South Asian countries. Economic Modelling, 35(C), pp. 654-660. doi: 10.1016/j.econmod.2013.07.040

Bhagwati, J. \& Srinivasan, T. (1979). Trade policy and development. En R. Dornbusch \& J. Frenkel (Eds.), International Economic Policy: Theory and Evidence. Baltimore: Johns Hopkins University Press.

Bhagwati, J. N. (1988). Poverty and public policy. World development, 16(5), 539-555.

Dollar, D., \& Kraay, A. (2007). Trade, Growth, and Poverty. En J. Clift \& E. Diehl (Eds.), Financial Globalization: The Impact on Trade, Policy, Labor, and Capital Flows (pp. 70-73). Washington, DC: International Monetary Fund.

Feder, G. (1983). On exports and economic growth. Journal of Development Economics, 12(1-2).

Fujii, G. \& Cervantes, R. (2013). México: Valor agregado en las exportaciones manufactureras. Revista CEPAL, (109), pp. 143-158.

Gabrielle, A. (2004). Exports of services and economic growth for developing countries. Trade and Development presentado en la conferencia de United Nations.

Giles, J. \& Williams, C. (2000). Export-led growth: a survey of the empirical literature and some non-causality results. Journal of International Trade and Economic Development, 9(3), pp. 261-337.

Gokmenoglu, K., Sehnaz, Z., \& Taspinar, N. (2015). The Export-Led Growth: A Case Study of Costa Rica. Procedia Economics and Finance, 25, pp. 471-477.

Grossman, G. M. \& Helpman, E. (1991). Innovation and Growth in the Global Economy. Cambridge: Massachusetts, MIT Press.

Hausmann, R., Hwang, J., \& Rodrik, D. (2007). What you export matters. Journal of Economic Growth, 12(1), pp. 1-25.

Helpman, E. \& Krugman, P. (1985). Market Structure and Foreign Trade. Cambridge: Massachusetts, MIT Press.

Instituto Nacional de Estadística y Geografía (INEGI). (2016). Tabla de Correlación entre la Tarifa de la Ley de los Impuestos Generales de Importación 
y de Exportación (TIGIE) y el Sistema de Clasificación Industrial de América del Norte (SCIAN). Recuperado de http://www3.inegi.org.mx/sistemas/ clasificaciones/tigie/tigie.aspx

Instituto Nacional de Estadística y Geografía (INEGI). (2017). Valor Agregado de Exportación de la Manufactura Global (VAEMG). Recuperado de https:// www.inegi.org.mx/temas/pibval/

Kohli, I. \& Singh, N. (1989). Exports and growth: critical minimum effort and diminishing returns. Journal of Development Economics, 30(2).

Krueger, A. (1980). Trade policy as an input to development. American Economic Review, 70(2).

Levy, N. (2015). El Neo-mercantilismo fallido en América Latina: la experiencia Mexicana de las últimas décadas. En Levy, Domínguez y Salazar (Coord.), Crecimiento económico, deudas y distribución del ingreso: nuevos y crecientes desequilibrios. Ciudad de México: UNAM.

Medina-Smith, E. J. (2000). Is the export-led growth hypothesis valid for developing countries? A case study of Costa Rica. New York: United Nations

Molina, D., Heuser, C., \& Mesquita, M. (2016). Infraestructura y desempeño de las exportaciones en la Alianza del Pacífico. Washington: Banco Interamericano de Desarrollo.

Moreno-Brid, J. C.; Rivas, J. C., \& Santamaría, J. (2005). Mexico: economic growth, exports and industrial performance after NAFTA. México, D.F.: Publicación de las Naciones Unidas.

Stiglitz, J. E. (2007). Making Globalization Work for Developing Countries. New York: Norton \& Company, Inc.

Thirlwall, A.P. (1979). The balance of payments constraint as an explanation of international growth rate differences. Quarterly Review, 32(128).

Yee, C. (2016). Export-Led Growth Hypothesis: Empirical Evidence from Selected Sub-Saharan African Countries. Procedia Economics and Finance, 35, pp. 232-240. doi: 10.1016/S2212-5671(16)00029-0 